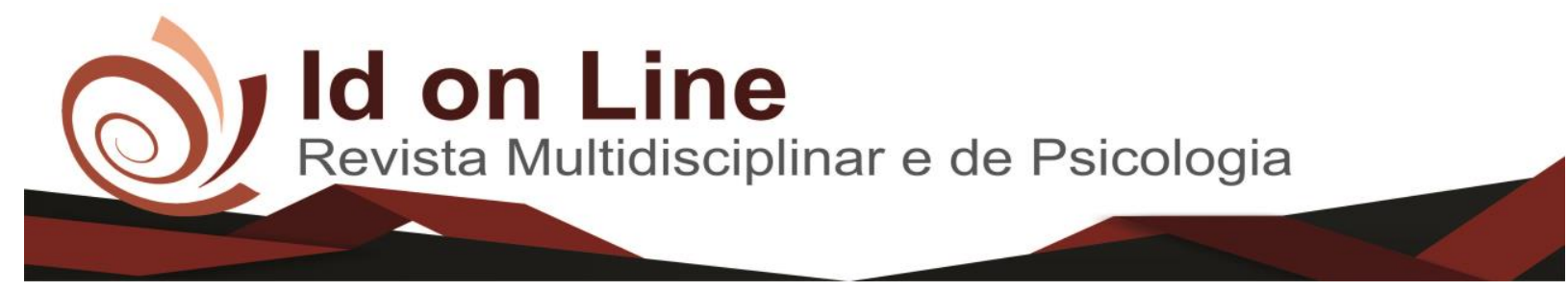

\title{
As Relações entre o Self/Eu estendido \\ e o consumo de marcas de Smartphones: um estudo com universitários da Região do Cariri cearense
}

\author{
Antônio Wilson dos Santos ${ }^{1}$; Antoniel dos Santos Gomes Filho ${ }^{2}$
}

\begin{abstract}
Resumo: Entendendo que o consumo não consiste apenas na aquisição física de bens/produtos e partindo dos indicativos empíricos e literários das ciências administrativas sobre as relações entre o self/eu estendido e o consumo de marcas de smartphones, o estudo em tela teve como objetivo identificar se os aparelhos celulares/smartphones produzem uma relação de extensão do self/eu em estudantes universitários da região do cariri cearense. Em relação aos itinerários metodológicos o estudo possui abordagem quantitativa exploratória, tendo como ferramentas de pesquisa a aplicação de um questionário online com base e adaptação na Self Brand Connection Scale, elaborada por Escalas e Bettman's (2003), cujo número amostral foi 40 participantes. Diante dos resultados e discussões do estudo considera-se que o grupo amostral sinalizou que a marca do celular/smartphone não é um indicativo "forte" de extensão do self/eu, sendo este produto percebido principalmente pela sua funcionalidade, porém há indicativos que uma parcela baixa da amostra possui relações mais intrínsecas com o celular/smartphone, sendo este representante uma possível extensão do self/eu. Esta baixa parcela amostral indica que há necessidade de ampliação nos estudos das relações de consumo e expansão do self/eu na Região do Cariri.
\end{abstract}

Palavras-Chave: Self/Eu Estendido. Consumo. Smartphone. Região do Cariri.

\section{The Relations between the Self and I extended and Smartphone Brands Consumption: A Study with University Students from the Cariri Region of Ceará}

\begin{abstract}
Understanding that consumption consists not only of the physical acquisition of goods / products and starting from the empirical and literary indicatives of the administrative sciences on the relations between the extended self and the consumption of smartphone brands, the study on screen had the objective to identify if mobile phones / smartphones produce a self extension relationship among university students from the region of the state of. In relation to the methodological itineraries, the study has a quantitative exploratory approach, using as research tools the application of an online questionnaire based and adapted in the Self Brand Connection Scale, elaborated by Escalas e Bettman's (2003), whose sample number was 40 participants. Given the results and discussions of the study, it is considered that the sample group signaled that the brand of the cellphone / smartphone is not a "strong" indication of extended self, being this product perceived mainly by its functionality, however there are indications that a low portion of the sample has more intrinsic relationships with the mobile
\end{abstract}

\footnotetext{
${ }^{1}$ Professor Diretor da Faculdade Vale do Salgado (FVS). Mestrando Profissional em Administração na Faculdade Boa Viagem (FBV). Especialista em Administração de Empresas e em Docência do Ensino Superior pelo Centro Universitário Dr. Leão Sampaio (UNILEÃO). Licenciado em Pedagogia pela Universidade Estadual Vale do Acaráu (UVA). E-mail: wilson@ @vs.edu.br

${ }^{2}$ Professor do curso de Administração da Faculdade Vale do Salgado (FVS). Mestre em Educação Brasileira pelo Programa de PósGraduação em Educação da Universidade Federal do Ceará, com área de concentração em História e Educação Comparada. Discente de Licenciatura em Filosofia na Faculdade Católica de Fortaleza (FCF). Coordenador do Laboratório Interdisciplinar em Estudos Organizacionais e do Trabalho (LIEOT/ FVS). Professor-pesquisador do Núcleo de Estudos Comparados em Corporeidades, Alteridade, Ancestralidade, Gênero e Gerações (NECA-GE/UFCA) e do Laboratório Interdisciplinar em Estudos da Violência (LIEV/UNILEÃO). Especialista em Gestão de Recursos Humanos pela Faculdade de Juazeiro do Norte (FJN). Tecnólogo em Gestão Comercial pelo Centro Universitário Dr. Leão Sampaio (UNILEÃO). Licenciado em Pedagogia pela Faculdade Kurios (FAK). E-mail: antonielsantos@fvs.edu.br / antoniel.historiacomparada@gmail.com
} 
phones / smartphone, this representative being a possible extension of the self. This low sample size indicates that there is a need for amplification in the studies of consumer relations and self expansion in the Cariri Region.

Keywords: Extended self. Consumption. Smartphone. Cariri Region.

\section{Introdução}

O consumo, numa perspectiva mais alargada, é algo constituinte da vida humana. Porém, é notório que a partir da revolução industrial no século XVIII na Europa iniciou-se uma série de transformações no mundo ocidental, aumentando assim as atividades produtivas bem como a disponibilidade de produtos antes restritos a uma pequena parcela da população.

A revolução industrial europeia foi responsável por uma série de mudanças que afetou todas as instâncias da sociedade, inclusive no que tange às relações de trabalho, família e consumo, gerando assim novas configurações sociais que se estenderam para todo o mundo ocidental, como ressalta Gomes Filho et. al. (2017).

Contemporaneamente, a rapidez das transações comerciais, a constante inovação dos bens e serviços e suas possibilidades de consumo em qualquer parte do globo, bem como empresas de atuação global, disponibilizam os mais variados produtos aos consumidores (REICHOW, 2015).

As experiências de consumo estão para além das meras necessidades de cunho biológico ou utilitário de sobrevivência. O consumo também agrega questões de ordem sociológica e psicológica do consumidor, como diz Philips (2003) o que consumimos define o que nós somos. Assim, os processos de identificação social e individual são também atravessados pelas atividades de consumo, principalmente em tempos onde se cria "[...] em grande escala a vontade crônica dos bens mercantis, o vírus da compra, a paixão pelo novo, um modo de vida centrado nos valores materialistas" (LIPOVETSKY, 2010). Assim, o consumo gera no indivíduo uma possibilidade de liberdade de escolha e felicidade no momento presente, diante de um bem, como é o caso dos smartphones, que acompanham praticamente todos os indivíduos na dita sociedade da informação.

O consumo de celulares no Brasil fica mais evidente quando se tem acesso a dados estatísticos produzidos nos últimos anos. O crescimento do consumo de celulares por brasileiros entre 2005 e 2011 foi de 107,2 \% (KLENIA, 2013). Esses números se sobressaem 
ainda mais quando comparados com dados mais recentes. Um breve relatório apresentado por Dino (2016) sobre o uso de celulares por brasileiros chama atenção. O relatório revela que os brasileiros já usam mais celulares do que computador pessoal para o acesso à internet. $\mathrm{O}$ autor relata que em 2015 o acesso à rede através da banda larga móvel foi de 191,8 milhões de acessos e apenas 25,4 milhões em banda larga fixa. O relatório aponta que em 2014 o Brasil já era o sexto mercado mundial em consumo de smartphone. Um dado importante é que para o jovem brasileiro o smartphone é o item mais importante a ser levado a um evento.

Em relação a este último dado, aponta-se que a cultura do consumo de celulares/smartphones na transição de uma sociedade da produção, para uma sociedade do consumo que se estende para além do ato de compra, torna-se um artefato importante na afirmação do indivíduo, neste sentido o consumo de celulares/smartphones perde sua simples função utilitária e ganha significados, identidades e dimensões sociais, pois ao comprar um celular/smartphone, os consumidores "[...] também estão comprando toda uma gama de significados simbólicos que expressam pertencimento ao mundo social [...]" (SILVA, 2007) e que pode funcionar como meio de afirmação identitários do usuário ou de um determinado agrupamento.

Há uma relação entre o consumidor e o objeto de consumo que vai além da simples aquisição do bem ou serviço. Compreender as relações consumidor-objeto é importante para entender o comportamento do consumidor. Segundo Belk (1988) não se pode avançar na compreensão do comportamento do consumidor sem que haja um entendimento mínimo sobre os significados que os consumidores dão aos seus bens e/ou posse. Para ele, as posses são partes do "eu", extensões, algo inseparável, por isso ao estudar esta relação estamos expandindo a compreensão do comportamento do consumidor.

Assim, quando pensamos nos celulares/smartphones, tanto fisicamente, como simbolicamente, mediado pela marca, pode influenciar diretamente na formação da identidade, bem como como extensão de seu self. O celular/smartphone conforme observou Lassen (2005, p.1) está sempre junto aos indivíduos, assim "os usuários possuem um relacionamento emocional com seus telefones e sentem-se ligados a eles.".

Diante destes apontamentos oriundos tanto da literatura especializada, como das experiências empíricas dos pesquisadores enquanto professores universitários emergiram os seguintes questionamentos: Como os universitários percebem a relação self/eu e o consumo e/ou posse de celulares/smartphones? Os celulares/smartphones proporcionam uma expansão 
do self/eu dos estudantes? Frente a estas indagações, o presente artigo tem como objetivo identificar se os aparelhos celulares/smartphones produzem uma relação de extensão do self/eu em estudantes universitários da região do cariri cearense, tendo como objetivos específicos, conhecer as relações e influências do consumo destes jovens, tanto individualmente como coletivamente, e descrever como as marcas podem tornar-se extensões do self/eu nas relações sociais. Consideramos que as questões levantadas neste estudo são de fundamental importância para as ciências administrativas, em especial para o marketing em sua dimensão holística, já que um dos principais objetivos do marketing "é desenvolver relacionamentos profundos e duradouros com todas as pessoas ou organizações que podem, direta ou indiretamente, afetar o sucesso das atividades da empresa." (KOTLER; KELLER, 2006, p.16), neste sentido as organizações empresariais necessitam conhecer melhor seus clientes, em diversas instâncias de suas vidas e comportamentos de consumo.

\section{O Consumo e o Self/Eu Estendido}

O momento do consumo de algum bem ou serviço é atravessado por uma série de questões que vão desde os meios sociais onde o consumidor está inserido, até mesmo pelo sentimento de encantamento e paixão pela posse de uma determinada coisa.

Como apontou Richins (1997) e Ahuvia (2005) há paixão muitas vezes relacionada aos momentos de consumo e as marcas disponíveis no mercado. Essa paixão ou vínculo forte entre os consumidores e as marcas de suas preferências é definido muitas vezes como uma fonte de desejo entre consumidor-objeto.

As marcas enquanto elemento simbólico vão além do vínculo e/ou engajamento emocional na relação consumidor-objeto. O consumo de determinadas marcas vão proporcionar experiências na vida do consumidor que podem ser determinantes em suas experiências sociais e individuais, promovendo assim um determinado comportamento de consumo, ao ponto de muitas vezes sentirem a ausência da marca quando ela não está disponível no mercado.

$\mathrm{Na}$ medida em que o consumidor interage com uma determinada marca, entra em contato com os atributos hedônicos e é exposto a diversas experiências, que vão desde a sua interação com o ambiente de compra, até sua experiência de consumo social (BACHA; 
SANTOS; STREHLAU, 2009), gerando assim um engajamento emocional criando um vínculo entre o consumidor e a marca, essa identificação determina em boa parte a paixão e a relação do consumidor com a marca (AHUVIA, 2005).

A paixão e o vínculo que o consumidor estabelece com determinadas marcas é resultado de sua identificação com as marcas de sua preferência. Todavia, a relação do consumidor com uma marca favorita transcende a paixão, ela conecta com a personalidade de seu possuidor e identifica-se com seu selfleu, essa extensão entre o consumidor e a marca provoca sentimento de ausência ou o sentimento de perda quando a marca não está disponível e idealização da personalidade da marca.

$\mathrm{Na}$ medida em que o consumidor se associa a marcas congruentes com seu self/eu, ele fortalece o seu próprio conceito de self/eu, pois a marca pode ser um meio pelo qual ele entende seu self/eu. Assim, há uma congruência da criação de valor de uma determinada marca no mercado e os aspectos da personalidade do consumidor, público alvo, assim o consumidor estende o seu conceito de self/eu sobre a imagem simbólica da marca. Os consumidores desejam e preferem marcas que refletem seus self/eu (BELK, 1988; SIRGY, 1982). As marcas reforçam o self/eu e segundo Belk e Askegaard (2003) à medida que o indivíduo se relaciona de forma significativa com as marcas de sua preferência, através do self/eu, ele pode expandir para novos domínios.

A compreensão de como os consumidores se comportam na escolha de suas posses só é possível segundo Belk (1988) se compreender a ligação dos consumidores com essas posses (físicas e simbólicas). As posses são instrumentos utilizados pelos consumidores para expressar, desenvolver e mediar suas identidades. Segundo o autor, o fator chave para que se tenha uma clara ideia do que uma posse representa para o consumidor, está no fato de compreender que o consumidor considera as posses como parte de si mesmo e faz isso consciente ou não.

As posses conectam os seus proprietários, cria laços (entre eles a paixão) e desempenha um papel fundamental na criação do self do indivíduo. Na medida em que possuímos, ainda que de forma simbólica, a identidade de algum modo pode ser reafirmada e/ou mediada através dos vínculos com bens de consumo, como carros, casas, aquisição de determinados serviços ou aquisição de aparelhos eletrônicos, tais como os smartphones, por exemplo. Essas conexões podem dar o senso de quem é o indivíduo, de onde ele vem e para onde vai na sociedade de consumo, como bem aponta Belk (1988). 
Esse vínculo entre o objeto possuído e seu possuidor é o que Belk (1988) chama de self/eu estendido. O self estendido representa que o indivíduo considera como seu e que de certa forma ao incorporar pessoas, objetos e lugares como sendo seu, de fato, está expandindo a sua identidade e aquilo que gostaria de ser.

O self/eu estendido não se limita apenas a posse de objetos e sua relação com o dono, mas tem relação com partes do corpo, processos psicológicos, ideias, pessoas, lugares, coleções e presentes (BELK,1988). Para o autor, entender como as posses afetam o bem-estar de quem as possui é essencial para compreender sua conexão com suas posses.

A teoria do self estendido está ligada ao consumo e ao comportamento do consumidor. É através da posse que o indivíduo projeta a sua identidade. Ao se identificar com a posse, na verdade, há uma assimilação dos significados por parte do indivíduo, estendendo seu self para o objeto possuído (PAZ, 2016; BACHA; SANTOS; STREHLAU, 2009; BECK ET. AL. 2009; BACHA; SCHAUM, 2011). Para os autores, o consumo representa e/ou expressa o self/eu, e para Belk (1988) "as posses são refletores da identidade" dos indivíduos. O estudo dessa relação é essencial para compreensão de como os objetos, pessoas e lugares agem no manuseio de seus "eus" ou representações na sociedade diante de suas relações com o consumo de determinadas marcas.

O consumo e a posse têm um significado especial para o indivíduo. Ao consumir, o consumidor estabelece uma relação emocional com suas posses, tornando-as partes integrais de sua identidade, uma parte de si mesmo, uma extensão de seu corpo e de sua personalidade. Essa ligação emocional que os consumidores passam a ter com suas posses é tão forte que segundo Belk (1988) a perda intencional ou não de um determinado objeto é percebida como uma perda ou redução de seu self e causa um grande sofrimento ao indivíduo.

A relação existente entre o self e a posse é central para a compreensão de vários comportamentos de consumo (COHEN, 1989; BELK 1988) e na forma como as posses são incorporadas ao self/eu estendido do consumidor, sendo de fundamental importância esse conhecimento por parte das organizações empresariais na atualidade. 


\section{Metodologia}

No que tange os aspectos metodológicos esta pesquisa possui uma abordagem quantitativa, descritiva e exploratória. Em seus parâmetros exploratórios a pesquisa utilizou a técnica de amostragem não probabilística e tendo como instrumento de coleta de dados primários um questionário online, disponibilizado na rede mundial de computadores entre os dias 01 e 05 de Julho de 2017, obtendo um total de 40 questionários respondidos (Amostra: $\mathrm{n}=40$ ). Foi utilizado para medir as relações entre self/eu estendido e o consumo de marcas de smartphones a Self Brand Connection Scale, elaborada por Escalas e Bettman's (2003) utilizada e validada em pesquisas sobre a temática, sendo esta adaptada para o estudo em tela.

Integra a Self Brand Connection Scale os seguintes questionamentos: (01) Esta marca reflete quem eu sou?; (02) Eu sinto uma conexão pessoal com essa marca?; (03) Eu uso essa marca para comunicar quem eu sou para outras pessoas?; (04) Eu considero essa marca como parte de mim?, e (05) Esta marca me representa bem?. Além destas indagações buscamos também conhecer alguns parâmetros socioeconômicos dos participantes da investigação. Assim, os dados obtidos via questionário foram analisados através do Microsoft Excel 2010, sendo apresentados juntamente com as discussões na próxima seção.

\section{Resultados e Discussões}

A tabela 01 apresenta uma descrição do perfil demográfico da amostra estudada segundo: o gênero, renda mensal, faixa etária, município residente e ao nível superior.

Tabela 01: Perfil demográfico da amostra

\begin{tabular}{l|c}
\multicolumn{1}{c|}{ Perfil demográfico da amostra } & $\begin{array}{c}\text { Amostra } \\
\mathrm{n}=40\end{array}$ \\
\hline Gênero & $\mathbf{\%}$ \\
\hline Masculino & 47,5 \\
\hline Feminino & 52,5 \\
\hline Outro & 0 \\
\hline Renda Mensal & $\mathbf{\%}$ \\
\hline Até 01 salário mínimo & 25 \\
\hline De 01 a 03 salários mínimos & 42,5 \\
\hline De 03 a 05 salários mínimos & 20 \\
\hline De 05 a 15 salários mínimos & 12,5 \\
\hline Mais de 15 salários mínimos & 0 \\
\hline
\end{tabular}




\begin{tabular}{l|c}
\hline Faixa etária & $\%$ \\
\hline Até 15 anos & 0 \\
\hline De 16 a 24 anos & 42,5 \\
\hline De 25 a 29 anos & 22,5 \\
\hline De 30 a 39 anos & 27,5 \\
\hline De 40 a 49 anos & 7,5 \\
\hline Mais de 50 & 0 \\
\hline Município residente & $\%$ \\
\hline Juazeiro do Norte & 55 \\
\hline Crato & 20 \\
\hline Barbalha & 10 \\
\hline Jardim & 2,5 \\
\hline Antonina do Norte & 2,5 \\
\hline Missão Velha & 2,5 \\
\hline Milagres & 2.5 \\
\hline Mauriti & 2,5 \\
\hline Iguatu & 2,5 \\
\hline Nível superior & $\%$ \\
\hline Graduação & 62,5 \\
\hline Especialização & 25 \\
\hline Mestrado & 7,5 \\
\hline Doutorado & 5 \\
\hline Pós-doutorado & 0 \\
\hline
\end{tabular}

Fonte: Dados da pesquisa (2017)

Como pode ser observado o perfil que sobressai é constituído por pessoas do gênero feminino, com renda mensal de 01 a 03 salários mínimos, com faixa etária entre 16 e 24 anos, residente no município de Juazeiro do Norte e graduado. Aponta-se que a concentração de pessoas residindo em Juazeiro do Norte se dá pelo fato do município ser considerado o maior polo universitário da região do cariri e interestadual, como aponta os dados disponibilizados pela gestão municipal:

O polo universitário de Juazeiro do Norte já conta com 56 cursos de graduação e ganhou notoriedade em nível de Nordeste. A influência das 10 Instituições de ensino superior, sendo três universidades e sete faculdades, é estimada sobre uma população de 2,5 milhões de habitantes do Cariri e estados vizinhos. Com mais de 20 mil estudantes, Juazeiro se tornou um grande polo universitário no interior cearense e um dos mais importantes do Nordeste (JUAZEIRO, 2017, s/p).

Diante desta questão observa-se que a maioria dos universitários $(62,5 \%)$ estão cursando uma graduação ou já concluíram. Esse parâmetro pode ser um indicativo sobre as formas de consumo, pois quando se observa a renda, vê-se que a maioria dos participantes está na Classe D, segundo a classificação do Instituto Brasileiro de Geografia e Estatística (MARKETING DIRETO, 2017). 
Como disse Belk (1988) os consumidores preferem marcas que refletem seus self/eu, pois o consumo de uma marca está correlacionado a personalidade do sujeito, e também as suas relações socioeconômicas, uma vez que a posse e o consumo tem um significado para o indivíduo. Assim, possuir um determinado smartphone pode ter várias significações para o grupo investigado, sendo que estes significados podem estar atravessados pelas questões de renda. A tabela 02 apresenta as marcas utilizadas pelos participantes da pesquisa e a faixa de preços destes smartphones.

Tabela 02: Marca e preço do smartphone

\begin{tabular}{l|c}
\multicolumn{1}{c|}{ Perfil demográfico da amostra } & $\begin{array}{c}\text { Amostra } \\
\mathrm{n}=40\end{array}$ \\
\hline Marca do Smartphone & $\boldsymbol{\%}$ \\
\hline Motorola & 37,5 \\
\hline Samsung & 22,5 \\
\hline Apple & 20 \\
\hline Positivo & 2,5 \\
\hline Nokia & 2,5 \\
\hline LG & 2,5 \\
\hline Asus & 2,5 \\
\hline Lenovo & 5 \\
\hline Multilaser & 2,5 \\
\hline Microsoft & 2,5 \\
\hline Faixa de preço do smartphone & $\%$ \\
\hline Até 299 R $\$$ & 5 \\
\hline De 300 a 599 R\$ & 10 \\
\hline De 600 a 899 R $\$$ & 22,5 \\
\hline De 900 a 1199 R $\$$ & 22,5 \\
\hline De 1200 a 1499 R\$ & 17,5 \\
\hline De 1500 a 1799 R\$ & 5 \\
\hline Mais de 1800 R $\$$ & 17,5 \\
\hline Fonte: Dado da pesquis (2017) & \\
\hline
\end{tabular}

Fonte: Dados da pesquisa (2017)

Como é visualizado na tabela 2, a maioria dos participantes da pesquisa utilizam como marcas de smartphone: Motorola (37,5\%), Samsung (22,5\%) e Apple (20\%), desembolsando entre 600 e 1199 R\$ (45\%) na aquisição de um aparelho. Como foi ressaltado por Ramalho e Ayrosa (2009) através da posse de um objeto o indivíduo projeta sua identidade. Neste sentido, nos chama atenção o número de $17,5 \%$ de participantes que desembolsaram mais de $1800 \mathrm{R} \$$ em um smartphone da marca Apple, na análise dos dados foi percebido que alguns participantes que utilizam essa marca estão na classe D e E. Essa inferência não é um indicativo de que as classes sociais com menor renda estejam consumindo marcas e produtos que outrora estavam restritas a alguns grupos sociais, ao contrário, é um indicativo que nos 
inquieta enquanto pesquisadores em relação à temática do self/eu estendido, desse modo surge a seguinte pergunta: Quais os significados do consumo de marcas com grande valor de aquisição por indivíduos de classes sociais menos favorecidas? Quais as relações com o self/eu estendido nestes casos específicos?

Apresentado o perfil demográfico da amostra e/ou participantes da investigação, neste momento centra-se na Self Brand Connection Scale, elaborada por Escalas e Bettman's (2003), como meio para identificar se os aparelhos celulares/smartphones produzem uma relação de extensão do self/eu em estudantes universitários da região do cariri cearense. Como foi apresentado na metodologia, a Self Brand Connection Scale consiste em cinco perguntas, deste modo, adiante apresentamos a tabela 03 referente e posteriormente teceremos uma análise sobre o objeto de estudo.

Tabela 03: Self Brand Connection Scale (Escalas; Bettman's, 2003)

\begin{tabular}{|c|c|c|c|c|}
\hline \multirow{4}{*}{ Self Brand Connection Scale } & \multirow{4}{*}{ Self Brand Connection Scale } & \multirow{2}{*}{\multicolumn{3}{|c|}{$\begin{array}{c}\begin{array}{c}\text { Amostra } \\
\mathrm{n}=40\end{array} \\
\text { Respostas predominantes } \\
\end{array}$}} \\
\hline & & & & \\
\hline & & Não & Sim & Complementos \\
\hline & & $\%$ & $\%$ & \\
\hline 01 & Esta marca reflete quem eu sou? & 87,5 & 12,5 & $\begin{array}{l}\text { "De certa forma sim, pois } \\
\text { é barata.". } \\
\text { "Para mim não. Para os } \\
\text { outros, às vezes, sim!". }\end{array}$ \\
\hline 02 & Eu sinto uma conexão pessoal com essa marca? & 82,5 & 17,5 & $\begin{array}{l}\text { "Talvez, pois ainda não } \\
\text { achei uma marca em que } \\
\text { me sentisse vinculada a } \\
\text { ela como vejo em muitos } \\
\text { colegas em relação a } \\
\text { algumas marcas, como a } \\
\text { Apple, por exemplo.”. } \\
\text { "Sentia, hoje não mais!". }\end{array}$ \\
\hline 03 & $\begin{array}{l}\text { Eu uso essa marca para comunicar quem eu sou para } \\
\text { outras pessoas? }\end{array}$ & 87,5 & 12,5 & $\begin{array}{l}\text { "Não. Uso somente por } \\
\text { necessidade!". }\end{array}$ \\
\hline 04 & Eu considero essa marca como parte de mim? & 92,5 & 7,5 & \\
\hline 05 & Esta marca me representa bem? & 87,5 & 12,5 & $\begin{array}{l}\text { "Não, apenas comprei } \\
\text { pois estava sem telefone, } \\
\text { e estava num preço } \\
\text { interessante, ou seja, mais } \\
\text { barato de todos.". } \\
\text { "Não. Meu celular é bom, } \\
\text { mas isso não quer dizer } \\
\text { que ele me representa.". }\end{array}$ \\
\hline
\end{tabular}

Fonte: Dados da pesquisa (2017) 
Diante dos dados obtidos através da Self Brand Connection Scale (ESCALAS; BETTMAN'S, 2003) e como disse Belk (1988) as posses de algum modo conectam seus proprietários e seus self/eu, assim o consumo pode ir além da simples aquisição de um bem ou serviço, representando um simbolismo. Mas na área de marketing é necessário que se pense na criação de valor para o consumidor, assim as necessidades podem se transformar em desejos de consumo. Em relação à indagação 01 é possível observar que para o grupo investigado as marcas de smartphones para estes indivíduos não refletem seus self/eu $(87,5 \%)$, é interessante perceber que no complemento de resposta que diz: "De certa forma sim, pois é barata.”, percebe-se que a marca está associada a possíveis fatores econômicos.

Dos participantes, $82,5 \%$ informaram que não sentem uma conexão com a marca do seu smartphone, e 17,5 apontou que de algum modo possui uma conexão com a marca. $\mathrm{O}$ seguinte complemento pode nos dar pistas para pensar sobre as pessoas que afirmam sentir-se conectadas a uma determinada marca de smartphone, como pode ser lido: "Talvez, pois ainda não achei uma marca em que me sentisse vinculada a ela como vejo em muitos colegas em relação a algumas marcas, como a Apple, por exemplo.”. O participante apontou que em suas relações sociais observa que algumas pessoas estão ligadas as marcas, como diz Richins (1997), o consumo de uma marca está atrelado a diversos sentimentos, entre eles a paixão por determinado bem, criando assim um comportamento de consumo da marca, acompanhada de um desejo e satisfação, que se estende além do individual.

Para a maioria dos participantes $(87,5)$ a marca do smartphone não serve como um meio de comunicação do self/eu em suas relações sociais, associado a esta questão ao observar as respostas da pergunta 04, pode-se inferir que para a amostra e/ou participantes o smartphone não atua simbolicamente como um self/eu estendido, pois há um indicativo que a escolha de uma marca de smartphone está ligada a sua funcionalidade e não a sua criação de valor simbólico, como pode ser visto na última pergunta, e no seguinte complemento: "Não, apenas comprei pois estava sem telefone, e estava num preço interessante, ou seja, mais barato de todos.". 


\section{Considerações Finais}

Diante dos procedimentos teórico-metodológico traçados para a investigação pode-se considerar que o grupo amostral investigado sinaliza que a marca do celular/smartphone não é um indicativo "forte" de extensão do self/eu, sendo este aparelho percebido principalmente pela sua funcionalidade, porém, há indicativos que uma parcela baixa da amostra possui relações mais intrínsecas com o celular/smartphone, sendo este representante uma possível extensão do self/eu. Frente a este último indicativo, aponta-se a necessidade de investigações que tomem esta parcela amostral de modo mais profundo, a fim de se reconhecer os pormenores das relações do self/eu estendido e o consumo de marcas de celular/smartphone. Assim, acredita-se que as pesquisas de abordagem qualitativas podem ser delineadas para tal fim, aumentando assim o escopo analítico do tema proposto neste estudo na Região do Cariri Cearense.

Outro ponto de destaque em relação ao grupo amostral refere-se ao reconhecimento da extensão do self/eu e o consumo de marcas de celular/smartphone em outros indivíduos. O reconhecimento das relações entre posse de um objeto e/ou marca de celular/smartphone por terceiros nos aponta para as questões simbólicas que envolvem o consumo, que como foi dito está para além da simples aquisição de um produto e/ou serviço.

A relação de ordem socioeconômica também aparece como destaque do grupo amostral, afinal como foi visto, mesmo a maior parcela do grupo dando indicativos que o consumo de celular/smartphone está correlato à necessidade do individuo versus a funcionalidade do produto, nota-se que alguns indivíduos em relação a sua renda mensal se dispuseram a pagar uma quantia elevada na aquisição de uma determinada marca de celular/smartphone. Como foi dito nos resultados e discussões, este fato não indica que indivíduos com menor renda não estejam consumindo produtos e serviços que outrora estavam restritos a pequenos grupos sociais, este fato é para nós apresentadores dos resultados do estudo em tela, inquietante. Assim, esperamos que as indagações surgidas no curso da análise desta investigação sejam promotoras de inquietação em outros investigadores/as no campo das ciências administrativas.

Como consideração "final", e reconhecendo os limites do estudo em tela, é pertinente que outros investigadores/as do campo das ciências administrativas e das demais ciências sociais aplicadas promovam outras pesquisas sobre a temática: relações de consumo e o 
self/eu estendido na Região do Cariri Cearense, como meio de conhecer as práticas e comportamentos do consumidor, bem como as possíveis estratégias empresariais no campo do marketing e da pesquisa mercadológica, já que tais temas são de profunda importância para o (re)conhecimento do mercado consumidor da Região do Cariri.

\section{Referências}

AHUVIA, A. C. Beyond The Extended Self: Loved Objects and Consumers' Identity Narratives. Journal of Consumer Research. v. 32, p. 171-184, 2005.

BACHA, M. L.; SANTOS, J. F. S.; STREHLAU, V. I. RENDA BAIXA: O SELF ESTENDIDO NA POSSE DO CELULAR. In: XII Semead: empreendedorismo e inovação, $2009 . \quad$ Disponível em: http://www.ead.fea.usp.br/semead/12semead/resultado/an_resumo.asp?cod_trabalho=667> . Acesso em. 30 Jun. 2017.

BACHA; M. L.; SCHAUM, A. A POSSE DE CELULAR E O SELF ESTENDIDO: UM ESTUDO LONGITUDINAL BASEADO EM ATITUDES DA BAIXA RENDA DE SÃO PAULO, BRASIL. In: Simpósio em tecnologias digitais e sociabilidades, Salvador, 2011.

BECK, C. G. et al. Meu Celular e Eu: mensurando a extensão do self. In: XXXIII Encontro da ANPAD, São Paulo, 2009.

BELK, R. W. Possessions and the extended self. In: Journal of Consumer Research, v. 15, n. 2, p. 139-168, Set. 1988.

BELK, R. W.; ASKEGAARD, S. The fire of disire: a multisider inquiry into consumer passion. In: Jornal of Consumer Research, v. 30, n. 3, 2003.

COHEN J. Na Over-Extended Self? In: Journal of Consumer Research. v. 16, n.1, p.-125$128,1989$.

DINO. Estatísticas de uso de celular no Brasil. 2016. Disponível em:< http://exame.abril.com.br/negocios/dino/estatisticas-de-uso-de-celular-no-brasildino89091436131/>. Acesso em: 09 Jun. 2017.

ESCALAS, J. E.; BETTMAN, J. R. You Are What They Eat: The Influence of Reference Groups on Consumer Connections to Brands. Journal of Consumer Psychology, v. 13, n. 3, p. 339-48. 2003.

GOMES FILHO, A. S. et. al. Relações de gênero e transformações das famílias: uma perspectiva sócio-histórica. In: MELO, M. A. S.; GOMES FILHO, A. S.; QUEIROZ, Z. F. 
(Orgs.). In: Epistemologias em confronto no direito: reinvenções, ressiginificações e representações a partir da interdisciplinaridade. Curitiba: CRV, 2017.

JUAZEIRO. Polo acadêmico. 2017. Disponível em: < http://www.juazeiro.ce.gov.br/Cidade/Polo-academico/ >. Acesso em: 20 Abr. 2017.

KLENIA. N. IBGE: uso de celular e internet cresceu mais de $100 \%$ no Brasil em seis anos. Tecmundo. Disponível em: <https://www.tecmundo.com.br/brasil/39797-ibge-uso-de-celulare-internet-cresceu-mais-de-100-no-brasil-em-seis-anos>: Acesso em: 09 Jun. 2017.

KOTLER, P.; KELLER, K. L. Administração de marketing. 12. ed. São Paulo: Pearson Prentice Hall, 2006.

LASSEN, A. Understanding mobile phone users and usage. Vodafone Group, 2005.

LIPOVETSKY. Gilles. A felicidade paradoxal: ensaio sobre a sociedade de hiperconsumo. São Paulo: Editora Schwarcz LTDA, 2010.

MARKETING DIRETO. Lista de classe sociais IBGE. 2017. Disponível em: < http://www.datosmarketing.com.br/listas-detalhes-classes-sociais.asp >. Acesso em: 29 Jun. 2017.

PAZ, E, C. O Gerenciamento de impressões e o Eu estendido: um estudo netnográfico sobre o consumo de viagens a partir de uma rede social virtual. Dissertação (Mestrado em Ciências Administrativas) - Universidade Federal do Pernambuco, Programa de em Administração, Recife-PE, 2016.

PHILLIPS, C. How do consumers express their identity through the choice of products that they buy? In: University of Bath School of Management Working Paper Series, v.17, p. 120, 2003.

RAMALHO, R.; AYROSA, E. A. T.; Subcultura Tuning: a identidade estendida na personalização de automóveis. In: RCA, Revista de Ciências da Administração - UFSC. v.1, n.24, 2009.

REICHOW, L. D. K. Comprar para ser: uma análise da transcendência das marcas na atualidade. Anais do Congresso ANPTECRE, v. 05, 2015.

RICHINS, M. L. Measuring Emotions in the Consumption Experience. In: Journal of Consumer Research, February, 1997.

SILVA, S. R. "EU NÃO VIVO SEM CELULAR": Sociabilidade, Consumo, Corporalidade e Novas Práticas nas Culturas Urbanas. In: Intexto, Porto Alegre: UFRGS, v. 2, n. 17, p. 1-17, 2007. 
SIRGY, M. J. Self-Concept in Consumer Behavior: A Critical Review. In: Journal of Consumer Research. V. 9, n. 3, p. 287-300, 1982.

\section{Como citar este artigo (Formato ABNT):}

SANTOA, Antônio W. dos; GOMES FILHO, Antoniel dos S. As Relações entre o Self/Eu estendido e o consumo de marcas de Smartphones: um estudo com universitários da Região do Cariri cearense. Id on Line Revista Multidisciplinar e de Psicologia, 2017, vol.11, n.38, p.42-56. ISSN: 1981-1179.

Recebido: 22.08.2017

Aceito: 06.10 .2017 